\title{
The Open AUC Project
}

\author{
Helmut Cölfen · Thomas M. Laue • Wendel Wohlleben · Kristian Schilling • \\ Engin Karabudak • Bradley W. Langhorst $\cdot$ Emre Brookes $\cdot$ Bruce Dubbs • \\ Dan Zollars $\cdot$ Mattia Rocco $\cdot$ Borries Demeler
}

Received: 21 January 2009 / Revised: 24 February 2009 / Accepted: 28 February 2009 / Published online: 19 March 2009

(C) The Author(s) 2009. This article is published with open access at Springerlink.com

\begin{abstract}
Progress in analytical ultracentrifugation (AUC) has been hindered by obstructions to hardware innovation and by software incompatibility. In this paper, we announce and outline the Open AUC Project. The goals of the Open AUC Project are to stimulate AUC innovation by improving instrumentation, detectors, acquisition and analysis software, and collaborative tools. These improvements are needed for the next generation of AUC-based research. The Open AUC Project combines on-going work from several different groups. A new base instrument is described, one that is designed from the ground up to be an analytical ultracentrifuge. This machine offers an open architecture, hardware standards, and application programming interfaces for detector developers. All software will use the
\end{abstract}

AUC\&HYDRO 2008-Contributions from 17th International Symposium on Analytical Ultracentrifugation and Hydrodynamics, Newcastle, UK, 11-12 September 2008.

Electronic supplementary material The online version of this article (doi:10.1007/s00249-009-0438-9) contains supplementary material, which is available to authorized users.

H. Cölfen · E. Karabudak

Colloid Chemistry, Max-Planck-Institute of Colloids

and Interfaces, Research Campus Golm,

Am Mühlenberg, 14424 Potsdam, Germany

T. M. Laue - B. W. Langhorst

Department of Biochemistry and Molecular Biology,

University of New Hampshire, Durham, NH 03824, USA

W. Wohlleben

Polymer Physics Research, BASF SE,

67056 Ludwigshafen, Germany

K. Schilling

Nanolytics, Gesellschaft für Kolloidanalytik mbH,

Am Mühlenberg 11, 14476 Potsdam, Germany
GNU Public License to assure that intellectual property is available in open source format. The Open AUC strategy facilitates collaborations, encourages sharing, and eliminates the chronic impediments that have plagued AUC innovation for the last 20 years. This ultracentrifuge will be equipped with multiple and interchangeable optical tracks so that state-of-the-art electronics and improved detectors will be available for a variety of optical systems. The instrument will be complemented by a new rotor, enhanced data acquisition and analysis software, as well as collaboration software. Described here are the instrument, the modular software components, and a standardized database that will encourage and ease integration of data analysis and interpretation software.

\author{
E. Brookes $\cdot$ B. Dubbs $\cdot$ D. Zollars $\cdot$ B. Demeler $(\square)$ \\ Department of Biochemistry, \\ The University of Texas Health Science Center, \\ San Antonio, TX 78229, USA \\ e-mail: demeler@biochem.uthscsa.edu \\ M. Rocco \\ Istituto Nazionale per la Ricerca sul Cancro (IST), \\ 16132 Genova, Italy
}




\section{Introduction}

For over 75 years, analytical ultracentrifugation (AUC) has proven to be a powerful method for characterizing solutions of macromolecules, and an indispensable tool for the quantitative analysis of macromolecular interactions. There are approximately 400 XLI analytical ultracentrifuges in use worldwide. These instruments are distributed almost evenly between industrial settings, primarily in pharmaceutical, biopharmaceutical, and polymer companies, and academic research facilities associated with biochemistry, biophysics, molecular biology, and material science programs. AUC provides core information for over 350 scientific publications a year (source: SciFinder 2008, using "AUC" as the search term) with usage increasing steadily (436 references in 2008).

From 1950 to 1992, advances in AUC were developed largely by academic labs and commercialized by Beckman Instruments (now Beckman Coulter Inc.). The current analytical ultracentrifuge, the XLI, was developed in the mid-1980s by retro-fitting an existing high-speed preparative centrifuge with absorbance and interference optics (Giebeler 1992; Yphantis et al. 1994). Since 1992, only one new optical system, the fluorescence optics (Schmidt and Riesner 1992; MacGregor et al. 2004; MacGregor 2000; Kroe and Laue 2009) has been commercialized and no improvements to the existing optical systems have been commercialized. Alternative base instruments have been commercially available that are less versatile than the XLI due to their lower maximum rotor speeds (LumiSizer by LUM GmbH: 6,000 rpm; BI XDC by Brookhaven Instruments: 10,000 rpm; DC24000 by CPS instruments: 24,000 rpm) and unconventional sample holders (monosector cells or discs instead of multisector cells). While these machines marginally qualify as analytical centrifuges, they clearly demonstrate the expansion potential for innovative AUC designs, such as one-shot detection of concentration profiles (LumiSizer) or non-optical detection by X-ray absorption (XDC).

The utility and feasibility of new optical systems for AUC has been demonstrated by the user community for turbidity (Cantow 1964; Scholtan and Lange 1972; Müller 1989; Mächtle 1992, 1999a), low angle laser light scattering (Bhattacharyya 2006; Mächtle and Börger 2006), Raman scattering (Bhattacharyya 2006), the Tsvetkov optics (Lavrenko et al. 1999), and Schlieren detection (Lloyd 1974; Cölfen and Borchard 1994; Mächtle 1999b; Börger et al. 2004). Even though compromised by the XLI geometry, each of these optical systems provides useful, even unique ways to monitor sedimenting particles. However, they cannot be made available commercially due to the legal restrictions on the XLI.

The Open AUC Project will remedy this situation by providing a new, alternative analytical ultracentrifuge plat- form, open source software drivers to operate the instrument, open source data analysis and data management tools, and open hardware and software standards that will encourage innovation. The current status of these advances is described here.

\section{Why do we need a new ultracentrifuge? Problems and restrictions with detector development for the XL ultracentrifuge platform}

Since the introduction of the XL-I and the digitalization of experimental data, tremendous progress has been made in analysis software and method development. These software advances have enabled researchers to analyze AUC data with much higher quality than was available a decade ago. Sedimentation coefficient distributions (Stafford 1992; Schuck 2000; Demeler and van Holde 2004), molar mass distributions (Brookes et al. 2006; Brown and Schuck 2006; Brookes and Demeler 2007, 2008), interaction constants (Cao and Demeler 2008; Stafford and Sherwood 2004; Schuck 1998), particle size distributions with Angström resolution (Cölfen and Pauck 1997) and the simultaneous determination of size and shape distributions from sedimentation velocity experiments (Brookes and Demeler 2006; Brookes et al. 2006) are examples of sophisticated data analysis methods that are used routinely by AUC laboratories. Powerful software packages that combine these methods, such as Ultrascan (Demeler 2005), Sedfit/Sedphat (Schuck et al. 1998; Vistica et al. 2004) and Sedanal (Stafford and Sherwood 2004) are available free of charge. In particular, the source code for UltraScan is licensed under the GNU Public License (GPL) (http://www.gnu.org/copyleft/gpl.html), making it possible for other groups to make improvements or advances. These analysis programs are supported by workshops.

In contrast to the tremendous advances made in data analysis, hardware development has not progressed significantly. The commercially available hardware is based on 20-year-old detector technology, and while new detection systems have been developed in research laboratories, they are not commercially available. One reason for this lag is the cost and effort associated with the development of an AUC detector. Designing the optical, mechanical, and electrical components, as well as writing reliable real-time data acquisition software, makes detector development challenging. However, the overriding causes of slow detector development for the AUC are the geometric and vacuum constraints of the XLI platform. Constructing the custom hardware is time consuming and expensive. Furthermore, the geometric constraints imposed by the XLI result in compromised optical performance. Worst of all, insurance and liability concerns have made it cost-prohibitive for 
Fig. 1 a Xl-heatsink with six additional holes (indicated by arrows) drilled in as optical channels or for mounting optical or electric vacuum feeds. b The same heatsink mounted in a XL preparative ultracentrifuge with multiwavelength detector. c Multiwavelength detector mounted on an XL-I heatsink
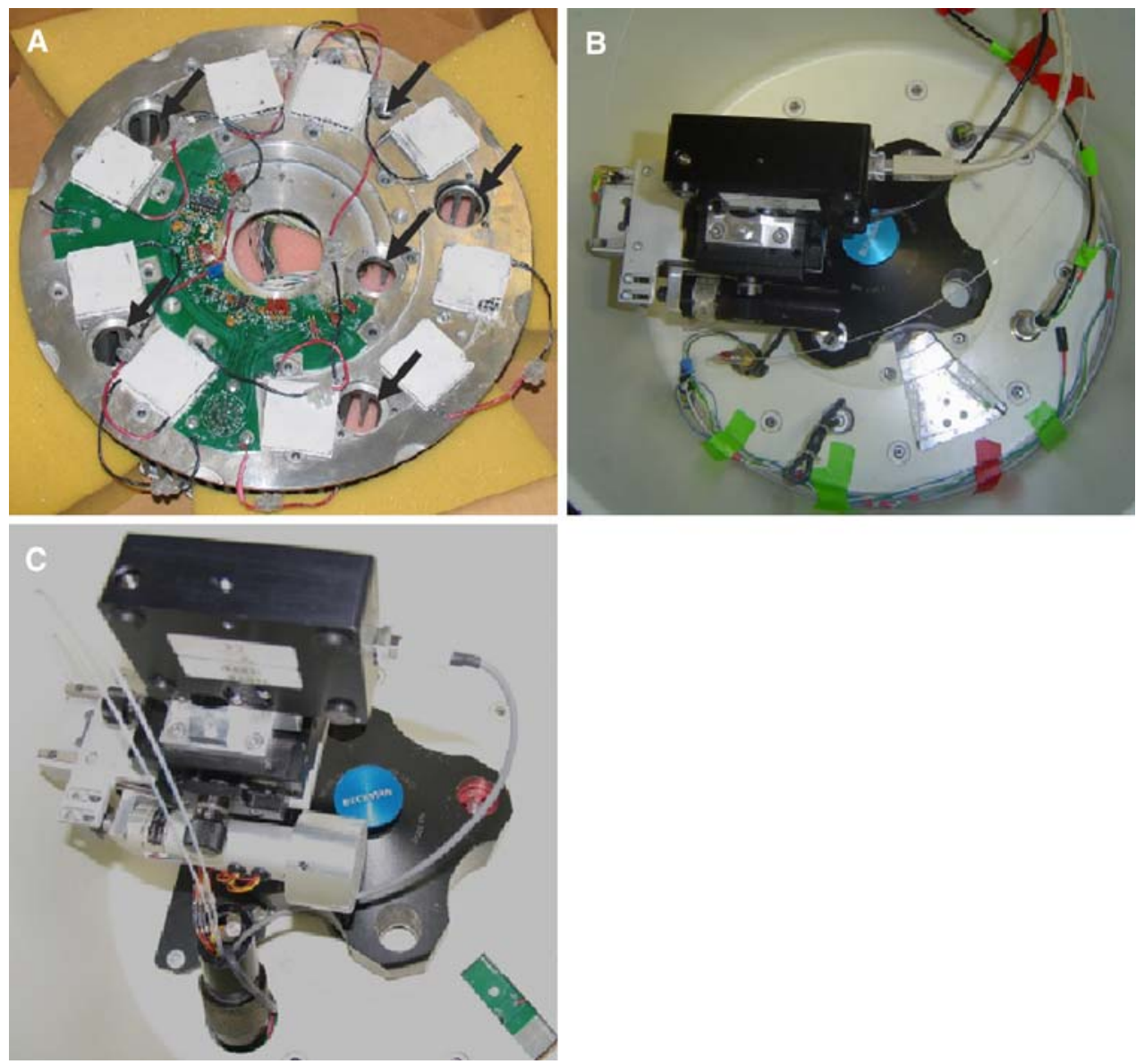

Beckman Coulter or other companies to commercialize any optics that require changes to the heatsink or in the vacuum/ containment chamber.

In most cases, a light source or its associated detector must be introduced into the vacuum chamber, which places severe material-compatibility constraints on components. The vacuum also poses significant cooling issues for lasers and mechanical positioners. Optical and electrical conduits, which require new holes in the heatsink (Fig. 1a), must be added to the vacuum chamber, creating warranty and safety issues for Beckman Coulter and the user (Fig. 1b). Although no problems have ever surfaced in machines with modified heatsinks, modification of a safety barrier in such an intrusive manner is certainly not desirable. To circumvent this problem, the standard XL-I heatsink may be converted for use with new optical systems (Fig. 1c). This heatsink has three holes, which can be used for conduits. As can be seen in Fig. 1c, the hole for the flash lamp and monochromator mount serves as cable and fiber conduit, leaving two more holes (photomultiplier mount and window in interference optical path) available for mounting a second and possibly a third optical system in the rotor chamber. Each of these additional optical systems, however, must fit in the remaining space. Furthermore, the introduction of new hardware in the vacuum chamber raises concerns about rotor explosion containment.

The XL-platform also suffers from a lack of publicly available software drivers to operate the instrument. System commands are issued to the XL via an RS232 serial port, but the command sequence is not published and has changed over the years. While the commands have been reverse-engineered (e.g. Aviv AU_AOS fluorescence detector operating software), the process is time consuming, and software development by a third party is very difficult.

From the above considerations, it becomes clear that the XL-platform is ill-suited for future AUC developments, and the requirement for an entirely new platform designed specifically for the analytical tasks of the AUC is apparent. The new AUC platform must:

1. Have open source communication protocols to allow for third party detector module development.

2. Provide mounts for light sources and detectors outside of the vacuum chamber and provide windows for all optical paths to illuminate the spinning AUC cell. This principle hearkens back to the Model E AUC series (1950-1972) by Beckman Instruments. 
3. Offer multiple optical paths and support simultaneous data acquisition by different optical systems.

4. Support a standard docking mechanism to allow for convenient swapping of multiple detector designs and allow the user to recombine those optical systems which may best meet the requirements for a particular application.

5. Offer the specialized hardware and software drivers needed to synchronize data acquisition with the spinning rotor. The hardware and software must be open source to encourage optical system development.

By meeting these specifications, the new AUC platform will encourage the development and commercialization of detectors. Because the difficult task of data acquisition synchronization is taken care of, and the open source communications modules relieve developers from writing software to operate the centrifuge, research laboratories will be able to develop new and specialized detectors. Likewise, it is hoped and anticipated that this "open platform" concept will encourage diverse optical instrument manufacturers to provide commercial detectors for the new AUC.

\section{Proposed hardware and software components}

\section{New ultracentrifuge}

A new analytical ultracentrifuge by Spin Analytical Inc. (Durham, NH), the centrifugal fluid analyzer (CFA), is being developed specifically for the Open AUC Project. This base instrument will facilitate significant improvements to the traditional AUC optical systems (absorbance, interference, and fluorescence) and, more importantly, stimulate the development of new optical detectors as outlined above. The CFA serves as a spinning sample holder (Fig. 2). All light sources and detectors are external to the rotor chamber. Three 50-mm diameter optical tracks are positioned at $120^{\circ}$ intervals around the rotor chamber. The larger diameter optics will overcome fundamental accuracy limits in the XLI Rayleigh optics (Yphantis et al. 1994). In addition, optical systems, which require multiple lenses and other optical components and thus a long optical path like the ultrasensitive Schlieren optics (Cölfen and Borchard 1994) can be realized. Optical working distances to the sample cells are minimized $(\sim 8-10 \mathrm{~cm}$, set by safety considerations) to optimize radial resolution.

The CFA uses the newest high-speed drive and drive electronics from Beckman Coulter. However, a different high-speed drive may be used by changing the base plate and drive electronics. The vacuum system is isolated from the rest of the instrument to reduce vibrations and surface contamination that degrade optical performance. The CFA does not require special electrical power and is mounted on a bench with space above and below it for optics.

Resources are provided on the CFA to aid optics development. Power $(5,15$, and 72 VDC) and a 48-bit external digital bus are provided on the CFA to operate the positioners, light sources, and detectors that comprise optical systems. A variety of specialized cards (described below) are being produced as part of the Open AUC Project to help interface optical systems to the signal bus. Since 48-bit digital I/O cards are widely available, developers can choose computers and operating systems that fit their requirements. There also is an internal data bus that provides the signals needed to synchronize external electronics to the spinning rotor (described below). Motion control may be provided by servos or by stepping motors. Descriptions, schematics, and timing diagrams for the bus signals will be published, as will the data bus and motion control driver software source code. While these resources are provided to help AUC developers, they are not constrained to use them.

\section{New rotor}

An eight-hole, fiber composite rotor that will operate at $60,000 \mathrm{rpm}$ is under development (Spin Analytical). A fiber composite rotor offers three advantages over titanium rotors. First, due to its lower mass and greater strength, fiber composite offers a higher safety margin than titanium. Furthermore, should a rotor failure occur, much less kinetic energy is released by the fiber composite than by a titanium rotor. Second, by using a stainless steel hub, a fiber composite rotor has a lower center of mass and moment of inertia, thus reducing precession and vibration compared to a monolithic titanium rotor. Third, a temperature measurement system may be incorporated directly inside the rotor, thus improving temperature accuracy. The design for the new rotor is shown in Fig. 2c.

\section{Optical systems for the CFA platform}

A number of optical systems should be adapted to the CFA platform. Traditional optical systems such as absorption and Rayleigh interference detectors should be improved to replace 20-year-old technology and take advantage of stateof-the-art equipment. In addition, several new detectors should be tested for suitability. The envisioned detectors and improvements for integration into the CFA are:

1. Absorbance optics Optical absorbance is the most widely used detector for AUC. The XLI absorbance system requires at least a minute to scan a single sample, and has notoriously poor wavelength reproducibility, limiting its utility for many applications. The prototype absorbance system built for the XLI uses a 
A

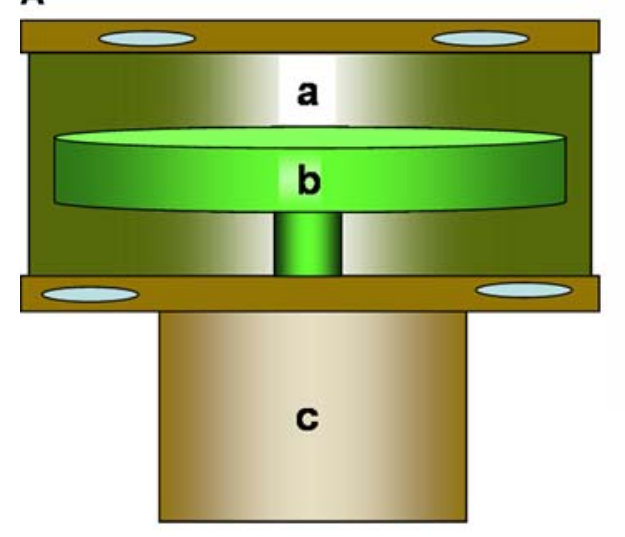

B

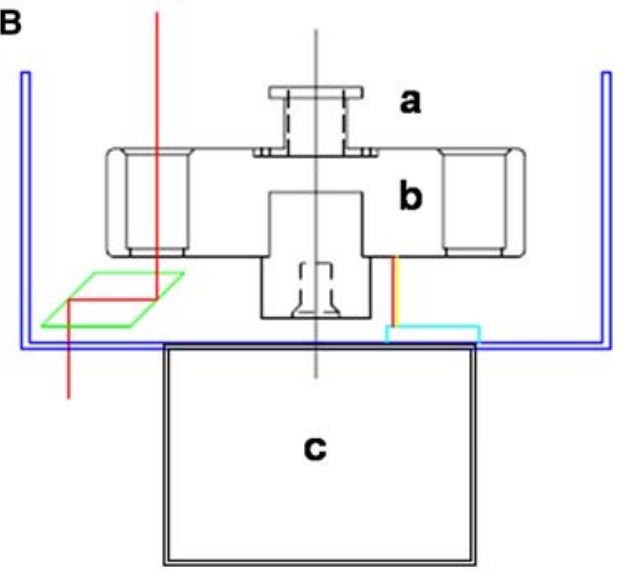

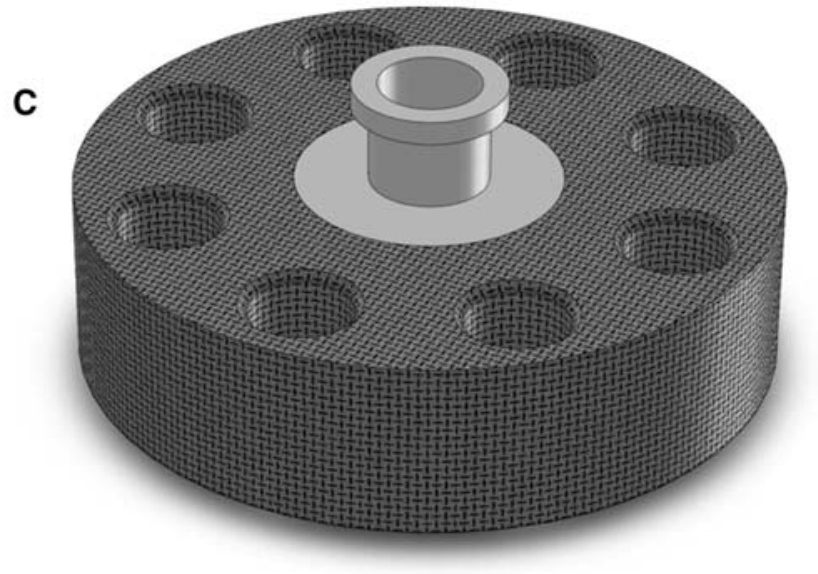

Fig. 2 The analytical ultracentrifuge for the Open AUC Project is simply a rotating sample holder that sits in three optical paths simultaneously. Sketches are shown of the CFA $(\mathbf{A}, \mathbf{B})$ and fiber composite eight hole rotor $(\mathbf{C})$ being developed for the Open AUC Project by Spin Analytical. The base centrifuge (A, B) simply consists of a vacuum containment chamber $(a)$, a rotor $(b)$, and high-speed motor $(c)$. Since only analytical rotors will be used in this instrument, a shorter rotor chamber may be used. Three optical tracks are arranged at $120^{\circ}$ intervals around the chamber. The optical tracks $(\mathbf{B})$ have to use a periscope to avoid the drive motor. While complicating the optical path somewhat, using the periscopes means that the CFA can accommodate different drive motors, thus providing flexibility for future designs. The new rotor $(\mathbf{C})$ being developed by Spin Analytical holds eight samples and may be operated at speeds up to $60,000 \mathrm{rpm}$. The sample holders are compatible with existing cell components continuous light source and software synchronization (Laue et al. 2006), acquires data from all samples simultaneously, has a 100-fold better wavelength reproducibility, has a tenfold higher sensitivity and precision, and acquires data at twice the radial resolution than the XLI.

2. Multi-wavelength absorbance optics The multi-wavelength system provides an absorbance spectrum at each radial position (Strauss et al. 2008; Bhattacharyya et al. 2006). Deconvolution of these spectra allows the discrimination of individual species sedimenting in complex mixtures of molecules. Cellular and molecular biology will benefit from this optical system since it will allow the size distributions of labeled molecules to be determined in a complex milieu (e.g. cell lysates). Molecules with different chromophores, such as DNA binding proteins, heme proteins or ligands and tagged molecules can be analyzed to study assemblies and complexes. For example, it will be possible to identify the components and characterize the cellular conditions under which molecules sediment as part of a macromolecular complex. Figure 3 shows the current multiwavelength detector and also the sedimentation of bovine serum albumin detected by two different operation modes: the time mode which detects the time dependent sedimentation of the sample with the detector set at a fixed radius and the radial mode which scans the cell radius at a given time. The optics will use a constant light source, a rapid scanning stepping motor, and a fast Andor ICCD camera capable of nanosecond integration time, allowing signal collection of all cell channels in each rotor revolution even at speeds as high as $60,000 \mathrm{rpm}$. 
Fig. 3 The multiwavelength detector arm mounted in an XLA AUC (left) as well as the typical experimental traces in time mode with radially fixed detector and radial mode scanning the AUC cell radially at a fixed time. The $x$-axis in these plots is the wavelength and the $z$-axis absorption. The data shown are for sedimenting bovine serum albumin $(B S A)$
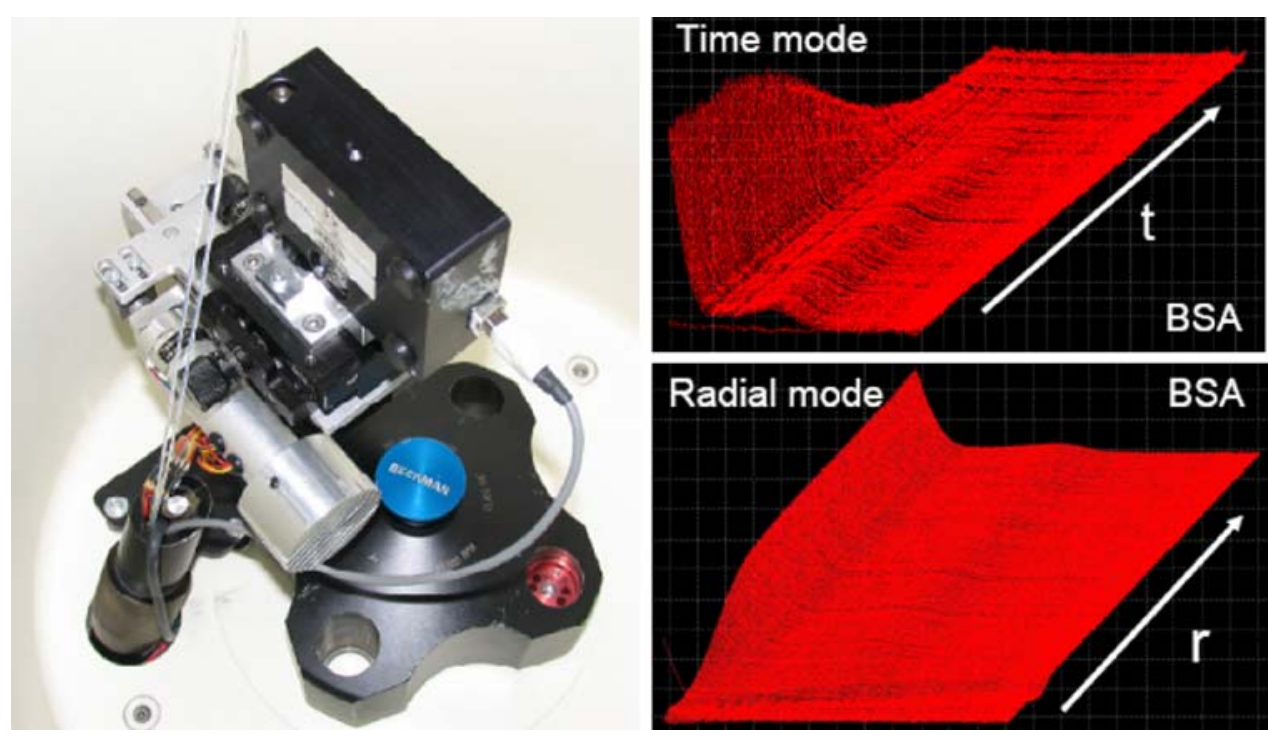

A
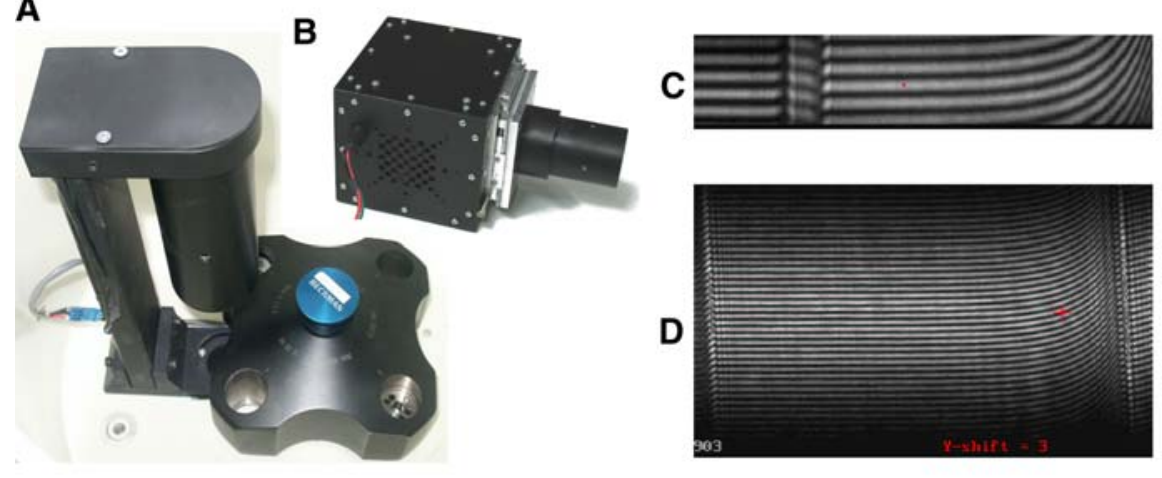

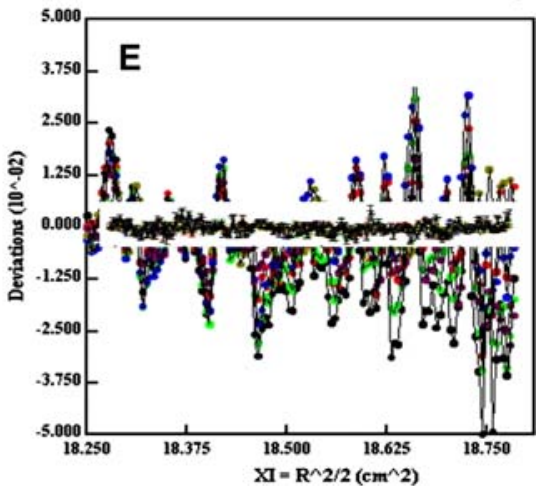

Fig. 4 Advances for Rayleigh Interference optics: a high-precision Rayleigh interference optical system mounted on an Xl ultracentrifuge (Laserarm from Spin Analytical); b large format interference camera from Philips $(3,000 \times 2,000$ pixels $)$; $\mathbf{c}$ data quality from the stock Beckman Coulter interference camera; $\mathbf{d}$ data quality from a large format Philips camera $(1,024 \times 2,048$ pixels $)$; e comparison of data quality as the residual noise from ten successive scans taken approximately $1 \mathrm{~min}$ apart. The residuals were calculated using WinMatch (available from http://www.rasmb.org) to optimize the fringe displacements. In c

3. High-precision interferometer Interference optics can detect solutes that have no convenient absorbance signal and have a higher precision than the absorbance optics. The XLI interferometer has a sensitivity of approximately 3.25 fringe $\mathrm{mg}^{-1} \mathrm{ml}^{-1}$ and a precision of \pm 0.01 fringe (Yphantis et al. 1994). Changes to the source and detector will improve the sensitivity more than twofold and the precision more than 100-fold (see detector shown in Fig. 4). The changes will extend the useful concentration range for interference detection by two orders of magnitude, making it useful for characterizing trace quantities of materials and high-affinity interactions (Howlett et al. 2006).

4. Low angle light scattering and turbidity Turbidity detectors have been described for AUC for particle size the residuals from the large format camera (rms 0.00017 fringe) are superimposed on those from the stock camera (camera rms 0.009). The increased number of fringes provided by the large format camera reduces the magnitude of the residuals over 50-fold, thus making it possible to acquire data at lower concentrations and improving the precision of analysis. Additional improvements can be expected from the Philips or any other large format CCD camera. Data supplied courtesy of David Yphantis and Jeff Lary

distribution analysis (Mächtle 1999a; Müller 1989; Scholtan and Lange 1972). A small-angle laser light scattering detector was also described to analyze molar mass distributions (Bhattacharyya 2006). These optical systems typically operate at a fixed radial position and acquire data with every rotor turn in order to capture the sedimentation of fast-moving particles. In combination with speed ramps they reach a measurement range of 20-5,000 nm for polymer particles (Mächtle and Börger 2006).

5. Multi wavelength fluorescence Fluorescence detection for AUC offers exquisite sensitivity and unparalleled solute discrimination for characterizing high-affinity interactions (Kingsbury et al. 2008) and the association state of proteins in serum and cell lysates (Kingsbury 
et al. 2008; Kroe and Laue 2009). The XLI AU-FDS fluorescence system is constrained to a single excitation wavelength and long-pass emission filter (MacGregor et al. 2004). A fluorescence system will be built that provides multiple excitation sources and four emission filters. This system will allow fluorescence and fluorescence resonance energy transfer characterization by AUC of multiple components and their complexes in biological fluids (e.g. serum, sputum, and cell lysates). Applications for these capabilities include detection of antigens and antibodies in biological fluids for medical research, and the direct physical characterization of complexes in proteomics research (Kroe and Laue 2009).

6. Schlieren optics Schlieren optics provide "tunable sensitivity," making them useful for working with extremely high-concentrations and with compressible fluids. The CFA version of Schlieren optics will be based on the prototype system built for the XLI (Mächtle 1999b), and will be useful for characterizing protein formulations (Shire et al. 2004). They also could be useful for the rapid, quantitative characterization of a variety of blood disorders (e.g. light chain amyloidosis, Sanchorawala 2006). However, the most important application of the Schlieren system will be analytical density gradients, which cannot be evaluated very well by any of the standard optical AUC systems (Mächtle and Börger 2006).

Operating software

The Open AUC Project includes software for operating the analytical ultracentrifuge. In order to accommodate diverse optical systems, the operating software is divided into "building block" modules that are interconnected using TCP/IP communications. The four building block modules that make up the operating software are: (1) machine services, (2) protocol services, (3) sample services, and (4) optical system operations (OSO). These modules are collectively called Analytical Ultracentrifuge-Advanced Operating System (AU-AOS).

1. The machine services module (MSM) controls centrifuge operation using ASCII commands that follow the XML-RPC standard. The draft standard for the commands is available http://www.rasmb.bbri.org/rasmb/ AOS/Open_AUC_Project/MachineServices/. Any computer with network connectivity may connect to and operate the CFA through TCP/IP communications.

2. The protocol services module (PSM) provides the information necessary to conduct an experiment, including the hardware description needed to operate optical detectors (sample holder angles and radii) and the method used (e.g. rotor speed, temperature, temperature tolerance, acceleration, braking, duration at each speed, any delay after reaching speed prior to data acquisition, time between data acquisition events and duration of the experiment) to collect the data. The identities of the protocol developer and user are kept with this information and saved in a database. A draft standard database structure is available (Langhorst 2008) and is described in "Proposed data analysis and data management infrastructure".

3. The sample services module (SSM) includes information about the solvent and solute properties, and the calculations needed to interpret AUC results (Langhorst 2008; Laue et al. 1992). Both a desktop and a web-served version of this module will be available.

4. Optical system operations: Separate modules are required to run the hardware for the different optical systems. These will be written using the Open AUC Project standards for communications with the Protocol services and Data handling services. The TCP/IP communications provide relatively low-speed communications, meaning that any time-critical code must be contained within the OSO modules for a specific optical system.

\section{Optical system interfaces}

The CFA and the Open AUC Project make rapid optical system development possible. The following specialized circuits and driver software will be available for optics developers. With these tools new optical systems (e.g. Raman, low angle X-ray scattering) can be developed for AUC.

1. Rotor timing pulse and master clock All data acquisition must be synchronized to the spinning rotor, and a rotor timing pulse with jitter $<1$ part in 4,000 is critical (Laue et al. 1984). The CFA meets this specification, and a digital rotor timing pulse is provided on the CFA signal bus. The master clock automatically provides $500 \mathrm{kHz}$ pulses below 7,000 rpm and $4 \mathrm{MHz}$ above $7,000 \mathrm{rpm}$ (with hysteresis to avoid switching back until 4,000 rpm). After each rotation, the period of rotation is latched and made accessible on the CFA signal bus, as is whether the low frequency or high frequency clock is being used (Laue et al. 1984).

2. Synchronizer The synchronizer uses the master clock to modulate either a light source or the detector so that data acquisition starts at a constant fraction of a revolution (the delay) and occurs over some fraction of a revolution (the duration), while compensating for propagation delays. The Open AUC Project synchronizer handles these functions using settings supplied by 


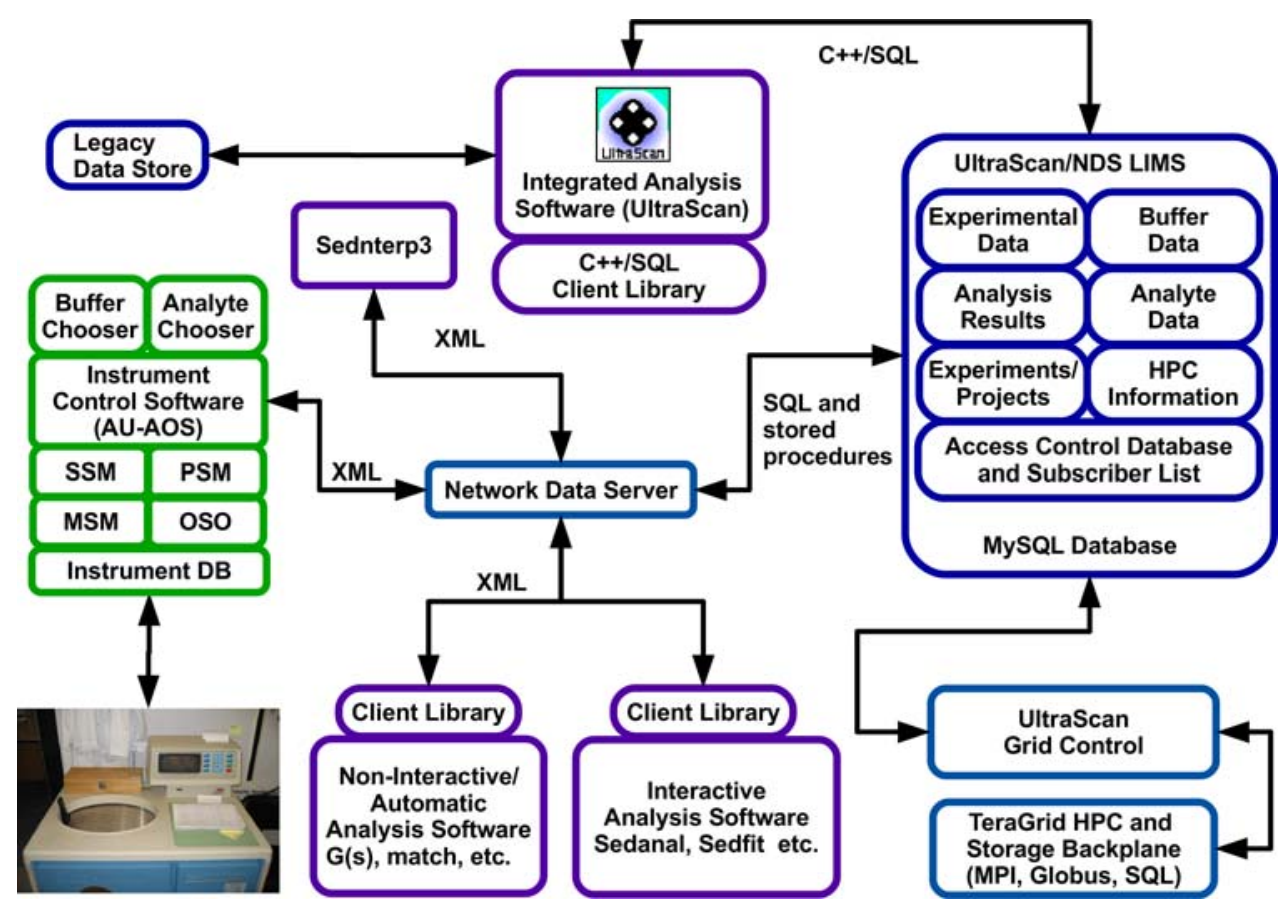

Fig. 5 Proposed Open AUC data analysis and data management infrastructure: shown are the UltraScan data analysis and LIMS database, the supercomputer module, the AU-AOS data collection and instrument operating system containing the sample service module (SSM), the protocol service module (PSM), machine service module (MSM), and the optical system operations (OSO), and tools for the integration of legacy data. The network data server provides an XML interface

an external computer on the CFA signal bus (Laue et al. 1984).

3. Digital data acquisition and data storage A board with a 14-bit $5 \mathrm{MHz}$ A/D with an addressable 16-bit by $4 \mathrm{Mb}$ buffer storage is available for the CFA signal bus. Digitizing may be gated externally (e.g. by synchronizer delay/duration functions). Data are stored sequentially in memory. Additional memory boards may be cascaded to provide up to 15 banks of memory to allow continuous data acquisition over long periods, such as required by the light scattering detectors (Mächtle 1999a). Memory may be accessed from the external computer over the CFA signal bus.

\section{Proposed data analysis and data management infrastructure}

The data analysis and data management components represent an integral cornerstone of the Open AUC Project. These elements will provide all required data analysis routines and provide Internet-capable database access for efficient data exchange, storage and management, and interface with the AU-AOS operating system to assist with data collection. Furthermore, a data interchange module between the AU-AOS and the LIMS system, and facilitates LIMS access by third party data analysis and interpretation software. Sednterp3 is a solution definition and calculation program. The US LIMS component stores experimental data, results, analyte information, the subscriber list, and other ancillary data. The UltraScan grid control module is responsible for managing analysis queues on the Teragrid infrastructure and communicating results with the US LIMS

provides a platform independent XML interface between database, data acquisition operating system and third party data analysis software. The system is based on the existing open source UltraScan data analysis platform (http://www. ultrascan.uthscsa.edu) and the UltraScan Laboratory Information Management System (US LIMS, http://uslims. uthscsa.edu). An overview of the proposed structure is shown in Fig. 5.

UltraScan data analysis

The UltraScan platform (Demeler 2008) provides an ideal open source environment for further development of the Open AUC Project. It is based on platform independent and portable development tools and open source programming languages (Qt, qwt, qwtplot3d, GNU C/C++, Perl, PHP, HTML, MySQL). UltraScan has been placed under Subversion (http://subversion.tigris.org) version control, providing support for multi-user development and branching. A Trac bug tracking system and WiKi (http://trac.edgewall.org) is used to manage development goals and user feedback via a ticketing system (http://wiki.bcf.uthscsa.edu/ultrascan/). UltraScan has been successfully ported to Windows (Windows 2000, NT4, XP, Vista), Linux/X11 (all hardware platforms), Macintosh OS-X and Darwin/X11 (Intel and 
G3/G4), Sun Solaris (7-10), SGI Irix (6.4 and higher), Open-, Net-, and Free-BSD, as well as other X11-based Unix versions.

UltraScan itself consists of an extensible $\mathrm{C}++$ class library and a Qt-based multi-threaded graphical user interface (GUI) with independent binaries linking to the UltraScan class library. This system has proven to be the best arrangement to guarantee optimal memory management and robustness. Should multiple modules be running and one of them encounters a fatal error, all other open modules are unaffected and will continue to function normally. The library offers classes for many popular data analysis methods such as van Holde-Weischet (Demeler and van Holde 2004), $\mathrm{d} c / \mathrm{d} t$ (Stafford 1992), second moment (van Holde 1985; Demeler 2005), $c(s)$ (Schuck 2000), nonlinear and linear least squares optimization (Demeler and Saber 1998), modeling of ASTFEM solutions of the Lamm equation (Cao and Demeler 2005, 2008) by two-dimensional spectrum analysis (Brookes et al. 2006, 2009), by genetic algorithm optimization (Brookes and Demeler 2006, 2007), and by Monte Carlo analysis (Demeler and Brookes 2008).

We propose a binary data standard for all experimental AUC data that replaces the currently used inefficient ASCII storage. An intrinsic absorbance spectrum can be derived by globally fitting wavelength scans taken at multiple concentrations. The spectra can be used as basis functions in the spectral decomposition fitting of spectra from mixtures (Demeler 2005). UltraScan further offers a complete data editing environment for absorbance, intensity, fluorescence, Rayleigh interference, and multiwavelength absorbance velocity and equilibrium data, and contains a module for import of legacy data and for conversion of binary formatted experimental data back to the legacy ASCII format. Intensity data can be converted to pseudo-absorbance data and corrected for time invariant noise contributions with the two-dimensional spectrum analysis.

A toolkit for visualization provides comprehensive twodimensional and three-dimensional visualization capabilities. A reporting mechanism dynamically generates HTML reports from an experiment for all analyses performed by the user. Hydrodynamic correction routines automatically adjust hydrodynamic data for standard conditions by calculating buffer densities and viscosities from user-supplied composition, as well as estimating partial specific volumes from protein sequence data.

A wide range of simulation modules assist the user in the design of experiments and modeling of experimental results. Multi-speed velocity and equilibrium experiments can be simulated with arbitrary precision, and for arbitrary models involving reversible self- and hetero-associating reactions. Kinetic rate constants can be defined to simulate the reaction kinetics. A new bead modeling program,
US-SOMO (based on SOlution MOdeler, Rai et al. 2005), facilitates rigid body modeling of NMR and X-ray crystallography structures (described below).

\section{Bead modeling}

The US-SOMO approach, fully described in another article in this issue (Brookes et al. 2009), is based on building a direct correspondence between groups of atoms within each residue in a biomacromolecule and the beads used to represent them. The bead models can be used to estimate the translational diffusion coefficient, the sedimentation coefficient, Stokes radius, rotational correlation time, intrinsic viscosity, and radius of gyration for comparison with results from hydrodynamic experiments and other related techniques. The bead models can also be used where the beads serve as scattering centers for the simulation of small-angle X-ray and neutron scattering data. US-SOMO generates a model of a macromolecule as an ensemble of rigid, non-overlapping spheres (beads) of different radii, utilizing a very well developed computational approach to calculate the hydrodynamic parameters (reviewed in García de la Torre and Bloomfield 1981; Spotorno et al. 1997; Carrasco and García de la Torre 1999). For instance, amino acids in proteins are usually represented with two beads, one for the atoms of the main chain and one for those of the side chain. The beads initial volumes are determined by the volumes of the atoms assigned to each bead and the volume of the theoretically bound water of hydration (Kuntz and Kauzmann 1974). Their position is determined by rules outlined in Rai et al. (2005), and several options are available to remove the bead overlaps while maintaining as much as possible the original surface envelope (Rai et al. 2005; Brookes et al. 2009). To improve the accuracy of the computations and reduce the computational load, an accessible surface area scan is performed on the original structure, identifying buried and exposed residues. Only the beads representing the exposed residues are used in the hydrodynamic computations. The residue definitions and their associate parameters reside in user-modifiable tables, affording a great flexibility in modeling. The program loads structures from protein data bank (PDB; Berman et al. 2000) formatted files, recognizing properly coded residues, and prompting the user when new residues are encountered. Currently, 64 residues containing $\sim 300$ different atom types are defined in the US-SOMO tables, including all standard amino acids, ribo- and deoxyribonucleosides and nucleotides, carbohydrates, and several co-factors. The program uses dynamic memory allocation and the size of the structure is theoretically limited only by the available memory in the computer. The original structures and the generated bead models can be visualized using the integrated molecular visualization program RasMol (Sayle and 
Milner-White 1995; http://openrasmol.org/\#Software). Several extensions are planned within the Open AUC Project, including a mechanism to describe flexible structures and the application of grid procedures to treat very large structures and complexes.

\section{US LIMS features}

The US LIMS provides a user-friendly web portal to the data storage and supercomputer analysis methods. All routines are programmed in XHTML, PHP and Perl, and adhere to the W3C strict coding standard (http:// www.w3.org/TR/xhtml1) to assure platform and browser independence. Each user is authenticated to a MySQL database, a separate instance belonging to each participating institution. Users are given a permission level which determines their role in the system (i.e. administrator, user, collaborator, analyst), and determines which data in the database can be accessed by the user. Any user can choose to share selected datasets from their own data with any other users to facilitate collaborations. The LIMS further offers web forms for the entry of peptide and nucleic acid sequences, buffer composition, upload of ancillary image data, as well as a virtual laboratory notebook for the annotation of projects and a place to provide experimental details. An interface for the retrieval of experimental and ancillary data as well as data analysis results is available as well. The supercomputer interface is used to enter analysis parameters and submit experimental analysis projects to the supercomputer clusters. Database tables in the US LIMS collect analysis results from the supercomputer, including submission parameters so analyses can be repeated in case of a malfunction. One of several clusters can be chosen for submission of compute-intensive jobs, and a detailed job queue informs the users about the status of their jobs, and permits job resubmission or job canceling. Supercomputer statistics such as used CPU time and performance are stored in database tables to permit accounting of computing allocations. Data analysis results are stored as compressed archives, whose contents are parsed by a web application that automatically generates a dynamically coded HTML file which presents the results through a browser as a well organized web page containing links to visualization images, ASCII formatted spreadsheets, and data analysis result reports generated by UltraScan.

\section{UltraScan grid control}

A separate grid control system running on the Bioinformatics Core Facility server at the University of Texas Health Science Center at San Antonio manages all submissions and queuing of analysis requests to remote clusters using the Globus (http://www.globus.org) and TIGRE
(http://www.hipcat.net/Projects/tigre) toolkits and by communicating with all major queuing systems such as PBS (http://www.openpbs.org) and Sun GridEngine (http:// gridengine.sunsource.net). Analysis results are committed back to the LIMS where they can be retrieved for further processing and visualized with UltraScan. The grid control module is written in Perl and communicates with selected remote clusters to run MPI jobs (http://www.open-mpi. org/) and to coordinate PBS job queuing (Brookes and Demeler 2008).

\section{Network data server}

The network data server (NDS) system acts as a data broker to provide platform independent access to all experimental and LIMS data by translating all data structures into clearly defined XML structures for maximum flexibility and ease of use for third parties. The XML design permits encapsulation of experimental information, including binary encoded experimental data, results, and experimental details, thereby minimizing misinterpretation. XML's extensible nature and the design of these data structures allows for addition of new information without breaking existing client programs. The NDS system acts as a secured gateway between the AU-AOS and the LIMS component, and will also provide a documented, open interface for third party data analysis software to acquire data and store results. Any developer is also free to connect to the LIMS directly using standard SQL communications, bypassing the NDS.

\section{Data flow}

The investigator initiates a project by creating an account on the LIMS portal (if none exists) and entering a project description in the laboratory notebook and by submitting all ancillary data such as buffer composition, analyte properties, gel images, absorbance scans, etc. to the database. Subsequently, an experimental design is added to the notebook. Next, the operator sets up the experiment by directly linking all acquired AUC data from within the data acquisition software (AU-AOS) with the investigator's database entries for each sample and commences the data acquisition. Communication between the AU-AOS and the US LIMS database is brokered by the NDS module. At this point, each experiment has a unique identification and the investigator identity associated with it which will carry forward through all data processing, analysis, and results presentation. During data collection all experimental data are forwarded to the US LIMS for storage in an internal, compressed binary format for maximum efficiency. A separate data conversion module assists with the import of data in legacy formats into the US LIMS. An export module 
permits export of US LIMS data to legacy formats for import into existing third party analysis software. In the next step, the experimental data are edited by storing all necessary transformations (meniscus position, data range minimum and maximum, baseline corrections, etc.) in the LIMS database as a separate dataset for each channel. Third party software will be able to import experimental data, store analysis results, and query the LIMS via the NDS. Edited data can be further processed by either a supercomputer-based analysis or by local UltraScan analysis using a control file to store experiment, cell, and channel information relevant to the analysis. Each channel produces a number of result files which are uniquely named by the analysis software according to a predefined standard. All analyses belonging to a channel are then grouped into a compressed archive and stored in the results database tables. During offline operation, the data can be processed from the user's computer without the requirement of an Internet connection. When Internet connectivity is again available, the results can be synchronized with the contents of the database. The proposed database structure is shown in the electronic supplementary material (Figure SI 1).

\section{Expandability}

The UltraScan C++ library API is documented with the open source Doxygen documentation system (http://www. doxygen.org). The library is organized into multiple modules designed to address GUI functions, and a modular design aids in the integration of new routines and expansions of the software. Translation classes support internationalization for different languages and the Qt framework provides all needed programming features for a modern software product. The modular, object-oriented design permits transparent maintenance and extension of UltraScan modules.

\section{Conclusions}

The Open AUC Project presented here is a serious and timely attempt, driven by users, to advance the versatile and valuable AUC method from a 20-year hiatus of technological advancements to a state-of-the-art technology. Such development is crucial for the survival of this classical method, which even after more than 75 years of intense hydrodynamic research still offers room for the introduction of new experimental methods-multiphase osmotic pressure determination is just one recent example (Page et al. 2008). A significant obstacle to innovation has been the dependence of the user community on a single ultracentrifuge platform, which requires feeding of light and electronics into the vacuum chamber of the ultracentrifuge. To eliminate this obstacle, the Open AUC Project can now facilitate the development of a common open source hardware platform, detectors, and software, freely available to anyone. Once a CFA AUC has been acquired, detector modules can be added and exchanged by the user at will. The proposed modular and open source software architecture further encourages development and provides a clear pathway for innovation. Communication will be performed over TCP/IP communications, and all data can be saved in an efficient relational database. By adopting a GPL license, we can assure that the technology developed finds broad implementation and we can guarantee that any intellectual property will remain freely available to any user. Due to the modular design of the Open AUC Project, data from XLbased ultracentrifuges (Beckman Coulter) can also be integrated into the proposed Open AUC Project data handling and analysis structure. Thus, the Open AUC Project will be beneficial for any AUC user regardless if a CFA or XL-I is used as hardware platform. Any data acquired by the instrumentation will be made available in a portable ASCII format to guarantee data exchange compatibility with third party software.

Acknowledgments The development of the UltraScan, US LIMS and AU-AOS software is supported by the National Institute of Health Grant \# RR022200 (to BD and TML).

Open Access This article is distributed under the terms of the Creative Commons Attribution Noncommercial License which permits any noncommercial use, distribution, and reproduction in any medium, provided the original author(s) and source are credited.

\section{References}

Berman HM, Westbrook J, Feng Z, Gilliland G, Bhat TN, Weissig H, Shindyalov IN, Bourne PE (2000) The protein data bank. Nucleic Acids Res 28:235-242. doi:10.1093/nar/28.1.235

Bhattacharyya S (2006) Development of detectors for the analytical ultracentrifuge. PhD Thesis, University of Potsdam, Potsdam

Bhattacharyya SK, Maciejewska P, Börger L, Stadler M, Gülsün AM, Cicek HB, Cölfen H (2006) Development of fast fiber based UVVis multiwavelength detector for an ultracentrifuge. Prog Colloid Polym Sci 131:9-22. doi:10.1007/2882_002

Börger L, Lechner MD, Stadler M (2004) Development of a new digital camera setup for the online recording of schlieren optical pictures in a modified Beckman optima XL analytical ultracentrifuge. Prog Colloid Polym Sci 127:19-25

Brookes E, Demeler B (2006) Genetic algorithm optimization for obtaining accurate molecular weight distributions from sedimentation velocity experiments. In: Wandrey C, Cölfen $\mathrm{H}$ (eds) Analytical Ultracentrifugation VIII. Prog Colloid Polym Sci 131:78-82

Brookes E, Demeler B (2007) Parsimonious regularization using genetic algorithms applied to the analysis of analytical ultracentrifugation experiments. In: GECCO proceedings ACM 978-1-59593697-4/07/0007

Brookes E, Demeler B (2008) Parallel computational techniques for the analysis of sedimentation velocity experiments in UltraScan. Colloid Polym Sci 286:138-148. doi:10.1007/s00396-007-1714-9 
Brookes E, Boppana RV, Demeler B (2006) Computing large sparse multivariate optimization problems with an application in biophysics. In: Supercomputing '06 ACM 0-7695-2700-0/06

Brookes E, Demeler B, Rosano C, Rocco M (2009) The implementation of SOMO (SOlution MOdeller) in the UltraScan analytical ultracentrifugation data analysis suite: enhanced capabilities allow the reliable hydrodynamic modeling of virtually any kind of biomacromolecule. Eur Biophys J Biophys Lett (this issue)

Brookes E, Cao W, Demeler B (2009) A two-dimensional spectrum analysis for sedimentation velocity experiments of mixtures with heterogeneity in molecular weight and shape. Eur Biophys J (this issue)

Brown PH, Schuck P (2006) Macromolecular size-and-shape distributions by sedimentation velocity analytical ultracentrifugation. Biophys J 90(12):4651-4661. doi:10.1529/biophysj.106.081372

Cantow HJ (1964) Zur Bestimmung von Teilchengrössenverteilungen in der Ultrazentrifuge. Makromol Chem 70:130-149. doi:10. 1002/macp.1964.020700110

Cao W, Demeler B (2005) Modeling analytical ultracentrifugation experiments with an adaptive space-time finite element solution of the Lamm equation. Biophys J 89:1589-1602. doi:10.1529/ biophysj.105.061135

Cao W, Demeler B (2008) Modeling analytical ultracentrifugation experiments with an adaptive space-time finite element solution for multi-component reacting systems. Biophys J 95:54-65. doi:10.1529/biophysj.107.123950

Carrasco B, García de la Torre J (1999) Hydrodynamic properties of rigid particles: comparison of different modeling and computational procedures. Biophys J 76:3044-3057. doi:10.1016/S00063495(99)77457-6

Cölfen H, Borchard W (1994) Ultrasensitive Schlieren optical system. In: Bonner RF et al (eds) Progress in biomedical optics, vol 2136. SPIE Bellingham, Washington, $\mathrm{p} 307$

Cölfen H, Pauck T (1997) Determination of particle size distributions with angstrom resolution. Colloid Polym Sci 275:175-180. doi: $10.1007 / \mathrm{s} 003960050068$

Demeler B (2005) UltraScan: a comprehensive data analysis software package for analytical ultracentrifugation experiments. In: Scott DJ, Harding SE, Rowe AJ (eds) Modern analytical ultracentrifugation: techniques and methods. Royal Society of Chemistry, Cambridge, pp 210-229

Demeler B (2008) The UltraScan data analysis software for analytical ultracentrifugation experiments. Version 9.9. http://www.ultrascan.uthscsa.edu

Demeler B, Brookes E (2008) Monte Carlo analysis of sedimentation experiments. Colloid Polym Sci 286:129-137. doi:10.1007/ s00396-007-1699-4

Demeler B, Saber H (1998) Determination of molecular parameters by fitting sedimentation data to finite-element solutions of the Lamm equation. Biophys J 74:444-454. doi:10.1016/S0006-3495(98) $77802-6$

Demeler B, van Holde KE (2004) Sedimentation velocity analysis of highly heterogeneous systems. Anal Biochem 335:279-288. doi:10.1016/j.ab.2004.08.039

García de la Torre J, Bloomfield VA (1981) Hydrodynamic properties of complex, rigid, biological macromolecules: theory and applications. Q Rev Biophys 14:81-139

Giebeler R (1992) The optima XL-A: a new analytical ultracentrifuge with a novel precision absorption optical system. In: Harding SE, Rowe AJ, Horton JC (eds) Analytical ultracentrifugation in biochemistry and polymer science. Royal Society of Chemistry, Cambridge, pp 16-25

Howlett GJ, Minton AP, Rivas G (2006) Analytical ultracentrifugation for the study of protein association and assembly. Curr Opin Chem Biol 10:430-436. doi:10.1016/j.cbpa.2006.08.017
Kingsbury JS, Klimtchuk ES, Laue TM, Théberge R, Costello CE, Connors LH (2008) The modulation of transthyretin tetramer stability by cysteine- 10 adducts and the drug diflunisal: direct analysis by fluorescence-detected analytical ultracentrifugation. J Biol Chem 283:11887-11896. doi:10.1074/jbc.M709638200

Kroe RR, Laue TM (2009) NUTS and BOLTS: applications of fluorescence detected sedimentation. Anal Biochem (in press)

Kuntz ID, Kauzmann W (1974) Hydration of proteins and polypeptides. Adv Protein Chem 28:239-345. doi:10.1016/S0065-3233 (08)60232-6

Langhorst B (2008) A system for automating the interpretation of analytical ultracentrifuge data. PhD Thesis, University of New Hampshire

Laue TM, Yphantis DA, Rhodes DG (1984) Rapid precision interferometry for the analytical ultracentrifuge. III. Determination of period of rotation, frequency of rotation and elapsed time. Anal Biochem 143:103-112. doi:10.1016/0003-2697(84)90563-3

Laue TM, Shah BD, Ridgeway TM, Pelletier SL (1992) Computer-aided interpretation of analytical sedimentation data for proteins. In: Harding SE, Rowe AJ, Horton JC (eds) Analytical ultracentrifugation in biochemistry and polymer science. The Royal Society of Chemistry, Cambridge, pp 90-125

Laue TM, Austin JB, Rau DA (2006) A light intensity measurement system for the analytical ultracentrifuge. Prog Colloid Polym Sci 131:1-8. doi:10.1007/2882_001

Lavrenko P, Lavrenko V, Tsvetkov V (1999) Shift interferometry in analytical ultracentrifugation of polymer solutions. Prog Colloid Polym Sci 113:14-22. doi:10.1007/3-540-48703-4_3

Lloyd PH (1974) Optical methods in ultracentrifugation, electrophoresis and diffusion: with a guide to the interpretation of records. Clarendon Press, Oxford

MacGregor IK (2000) Fluorescence optics for the analytical ultracentrifuge. MS Thesis, University of New Hampshire, Durham

MacGregor IK, Anderson AL, Laue TM (2004) Fluorescence detection for the XLI ultracentrifuge. Biophys Chem 108:165-185. doi: $10.1016 / \mathrm{j}$. bpc.2003.10.018

Mächtle W (1992) Analysis of polymer dispersions with an eight-cellAUC-multiplexer: high resolution particle size distributions and density gradient techniques. In: Harding SE, Rowe AJ, Horton JC (eds) Analytical ultracentrifugation in biochemistry and polymer science. The Royal Society of Chemistry, Cambridge, pp 147-175

Mächtle W (1999a) High-resolution, submicron particle size distribution analysis using gravitational-sweep sedimentation. Biophys J 76:1080-1091. doi:10.1016/S0006-3495(99)77273-5

Mächtle W (1999b) The installation of an eight-cell Schlieren optics multiplexer in a Beckman optima XLI/XL analytical ultracentrifuge used to measure steep refractive index gradients. Prog Colloid Polym Sci 113:1-9. doi:10.1007/3-540-48703-4_1

Mächtle W, Börger L (2006) Analytical ultracentrifugation of polymers and nanoparticles. Springer, Heidelberg

Müller HG (1989) Automated determination of particle-size distributions of dispersions by analytical ultracentrifugation. Colloid Polym Sci 267(12):1113-1116. doi:10.1007/BF01496933

Page MG, Zemb T, Dubois M, Cölfen H (2008) Osmotic pressure and phase boundary determination of multiphase systems by analytical ultracentrifugation. ChemPhysChem 9:882-890. doi:10.1002/ cphc. 200700668

Rai N, Nöllmann M, Spotorno B, Tassara G, Byron O, Rocco M (2005) SOMO (SOlution MOdeler): differences between X-ray- and NMR-derived bead models suggest a role for side chain flexibility in protein hydrodynamics. Structure 13:723-734. doi:10.1016/ j.str.2005.02.012

Sanchorawala V (2006) Light-chain (AL) amyloidosis: diagnosis and treatment. Clin J Am Soc Nephrol 1:1331-1341. doi:10.2215/ CJN.02740806 
Sayle RA, Milner-White EJ (1995) RasMol: biomolecular graphics for all. Trends Biochem Sci 20:374-376. doi:10.1016/S0968-0004 (00)89080-5

Schmidt B, Riesner B (1992) A fluorescence detection system for the analytical ultracentrifuge and its application to proteins, nucleic acids, viroids and viruses. In: Harding SE, Rowe AJ, Horton JC (eds) Analytical ultracentrifugation in biochemistry and polymer science. The Royal Society of Chemistry, Cambridge, pp 176207

Scholtan W, Lange H (1972) Determination of particle-size distribution of latices with ultracentrifuges. Kolloid Z Z Polymere 250:782-796. doi:10.1007/BF01498571

Schuck P (1998) Sedimentation analysis of noninteracting and self-associating solutes using numerical solutions to the Lamm equation. Biophys J 75:1503-1512. doi:10.1016/S0006-3495(98) 74069-X

Schuck P (2000) Size-distribution analysis of macromolecules by sedimentation velocity ultracentrifugation and Lamm equation modeling. Biophys J 78:1606-1619. doi:10.1016/S0006-3495 (00)76713-0

Schuck P, MacPhee CE, Howlett GJ (1998) Determination of sedimentation coefficients for small peptides. Biophys J 74:466-474. doi:10.1016/S0006-3495(98)77804-X

Shire SJ, Shahrokh Z, Liu J (2004) Challenges in the development of high protein concentration formulations. J Pharm Sci 93:13901402. doi:10.1002/jps.20079

Spotorno B, Piccinini L, Tassara G, Ruggiero C, Nardini M, Molina F, Rocco M (1997) BEAMS (BEAds Modelling System): a set of computer programs for the generation, the visualization and the computation of the hydrodynamic and conformational properties of bead models of proteins. Eur Biophys J 25:373-384 (Erratum 26:417)

Stafford W (1992) Boundary analysis in sedimentation transport experiments: a procedure for obtaining sedimentation coefficient distributions using the time derivative of the concentration profile. Anal Biochem 203:295-301. doi:10.1016/0003-2697(92)90316-Y

Stafford WF, Sherwood PJ (2004) Analysis of heterologous interacting systems by sedimentation velocity: curve fitting algorithms for estimation of sedimentation coefficients, equilibrium and kinetic constants. Biophys Chem 108:231-243. doi:10.1016/j.bpc.2003. 10.028

Strauss HM, Karabudak E, Bhattacharyya S, Kretzschmar A, Wohlleben W, Cölfen H (2008) Performance of a fast fiber based UV/Vis multi-wavelength detector for the analytical ultracentrifuge. Prog Colloid Polym Sci 286:121-128

van Holde KE (1985) Physical biochemistry, 2nd edn. Prentice-Hall, Englewood Cliffs, p 114

Vistica J, Dam J, Balbo A, Yikilmaz E, Mariuzza RA, Rouault TA, Schuck P (2004) Sedimentation equilibrium analysis of protein interactions with global implicit mass conservation constraints and systematic noise decomposition. Anal Biochem 326(2):234256

Yphantis DA, Lary JW, Stafford WF, Liu S, Olsen PH, Hayes DB, Moody TP, Ridgeway TM, Lyons DA, Laue TM (1994) On line data acquisition for the Rayleigh interference optical system of the analytical ultracentrifuge. In: Schuster TM, Laue TM (eds) Modern analytical ultracentrifugation. Birkhäuser, Boston, pp 209-226 